Discussiones Mathematicae

Graph Theory 25 (2005) 1-xxx

\title{
DISTANCE COLORING OF THE HEXAGONAL LATTICE
}

\author{
Peter JACKO \\ Carlos III University \\ Department of Business Administration \\ Calle Madrid 126, 28903 Getafe (Madrid), Spain \\ e-mail: peter.jacko@uc3m.es \\ AND \\ StANisLAV JENDROL' \\ P.J. Šafárik University \\ Institute of Mathematics \\ Jesenná 5, 04154 Košice, Slovakia \\ e-mail: jendrol@kosice.upjs.sk
}

\begin{abstract}
Motivated by the frequency assignment problem we study the $d$ distant coloring of the vertices of an infinite plane hexagonal lattice $H$. Let $d$ be a positive integer. A $d$-distant coloring of the lattice $H$ is a coloring of the vertices of $H$ such that each pair of vertices distance at most $d$ apart have different colors. The $d$-distant chromatic number of $H$, denoted $\chi_{d}(H)$, is the minimum number of colors needed for a $d$-distant coloring of $H$. We give the exact value of $\chi_{d}(H)$ for any $d$ odd and estimations for any $d$ even.

Keywords: distance coloring, distant chromatic number, hexagonal lattice of the plane, hexagonal tiling, hexagonal grid, radio channel frequency assignment.
\end{abstract}

2000 Mathematics Subject Classification: 05C15, $05 \mathrm{C} 12$. 


\section{INTRODUCTION}

By the distance $\operatorname{dist}_{G}(u, v)$ of two vertices $u$ and $v$ in a graph $G$ we mean the number of edges of the shortest path joining them. Given a positive integer $d$, a $d$-distant $n$-coloring of a graph $G$ is an assignment $\varphi$ that assigns numbers from the set $\{1,2, \ldots, n\}$ to the vertices of $G$ in such a way that each pair of vertices within the distance $d$ have assigned different colors. Then the $d$-distant chromatic number $\chi_{d}(G)$ of a graph $G$ is the minimum number $n$ such that there is a $d$-distant $n$-coloring of the graph $G$.

The $d$-distant coloring of graphs has been introduced by Wegner [14] (see also [9, 10]). Many articles, e.g. [1, 3, 4, 7], have studied a special case, the distant colorings with condition at distance 2. Several papers have been devoted to distant colorings during the last decade, see for example $[2,6,7$, $8,12]$ and references therein.

Consider a natural partition of the plane to regular hexagons as a 3regular graph, called a hexagonal lattice. The hexagonal lattice is a significant planar graph, one of the three regular-polygons-tilings of the plane. Given a positive integer $d$, how many colors are necessary to color the vertices of the hexagonal lattice so that any two vertices within distance $d$ are of distinct color? This question naturaly arises from the Frequency Assignment Problem (see for example [5]), where the vertices of a graph correspond to transmitter locations and their colors represent frequencies of the channels. The transmitters that are "very close", cannot use the same frequency due to interference.

Let $[x]$ for any $x \in \mathbb{R}$ be an integer such that $x-\frac{1}{2}<[x] \leq x+\frac{1}{2}$.

Several authors have studied situations when the transmitters are spread over the plane regularly, since the Cellular concept has been introduced [11]. Recently it has been shown by Fertin, Godard and Raspaud [2] that the $d$ distant chromatic number of the square lattice $S$ is $\chi_{d}(S)=\left[\frac{1}{2}(d+1)^{2}\right]$. Similar result has been discovered for the triangular lattice $T$ by Ševčíková [13], where she proved that $\chi_{d}(T)=\left[\frac{3}{4}(d+1)^{2}\right]$.

The main result of this paper is the following theorem:

Theorem 1.1. The d-distant chromatic number $\chi_{d}$ of the hexagonal lattice $H$ is

(i) $\chi_{d}(H)=\left[\frac{3}{8}(d+1)^{2}\right]$ for every odd $d \geq 1$,

(ii) $\chi_{2}(H)=4, \chi_{4}(H)=11, \chi_{6}(H)=20$,

(iii) $\frac{3}{8} d^{2}+\frac{3}{4} d+2 \leq \chi_{d}(H) \leq\left[\frac{3}{8}\left(d+\frac{4}{3}\right)^{2}\right]$ for every even $d \geq 8$. 
We believe that the following is true

Conjecture 1.2. For every even $d \geq 2$ there is

$$
\chi_{d}(H)=\left[\frac{3}{8}\left(d+\frac{4}{3}\right)^{2}\right] .
$$

We will prove this theorem after introducing some necessary properties and system of coordinates in the hexagonal lattice.

The symbol $H$ will be used for the hexagonal lattice, symbols $V, E$ for the vertex-set and edge-set, respectively, of the lattice $H$, symbol $\operatorname{dist}(u, v)$ for the distance of any two vertices $u, v \in V$ and symbol $\chi_{d}$ for the $d$-distant chromatic number of a graph $H$ in this article.

\section{Characterization of the Hexagonal Lattice}

Notice that the hexagonal lattice $H$ is a bipartite graph. Any circle of six vertices from $V$ will be called an eye. Fix an arbitrary eye to be the central eye and denote $R_{1}$ its vertex-set. For any positive integer $k$ define a $k$-th ring $R_{k}$ as a subset of $V$ such that the $R_{k}$ contains a vertex $v \in V$ if and only if the distance between vertex $v$ and the central eye satisfies $2 k-3 \leq \operatorname{dist}\left(v, R_{1}\right) \leq 2 k-2$ (note that the distance $\operatorname{dist}\left(v, R_{1}\right)$ is defined to be the minimum distance between $v$ and any vertex belonging to the set $\left.R_{1}\right)$. Clearly, the system of sets $R_{k}$ is a partition of the vertex-set of the hexagonal lattice $H$.

We similarly denote the following sets:

$$
\begin{aligned}
& R_{k}^{-}=\left\{v \in V(H) ; \operatorname{dist}\left(v, R_{1}\right)=2 k-3\right\} \text { and } \\
& R_{k}^{+}=\left\{v \in V(H) ; \operatorname{dist}\left(v, R_{1}\right)=2 k-2\right\} .
\end{aligned}
$$

Thus, $R_{k}^{-} \cup R_{k}^{+}=R_{k}$. Note, that $R_{1}^{-}=\emptyset$.

Proposition 2.1. For any positive integer $k$, no two vertices from $R_{k}^{-}$are adjacent in $H$. Furthermore, one of the vertices adjacent to $v \in R_{k}^{-}$belongs to $R_{k-1}^{+}$and the other two belong to $R_{k}^{+}$.

Proposition 2.2. For $k \geq 2$, the set $R_{k}^{+}$contains exactly six disjoint pairs of adjacent vertices (called corner vertices). Moreover, if $v \in R_{k}^{+}$is a corner 
vertex, then one of the vertices adjacent to $v$ belongs to $R_{k}^{-}$, one belongs to $R_{k}^{+}$and the third vertex belongs to $R_{k+1}^{-}$. If $v \in R_{k}^{+}$is not a corner vertex, then two of the vertices adjacent to $v$ belong to $R_{k}^{-}$and the last one belongs to $R_{k+1}^{-}$.

From the two listed propositions we immediately get the following Lemma.

Lemma 2.3. For any positive integer $k$ is $\left|R_{k}^{-}\right|=6(k-1)$ and $\left|R_{k}^{+}\right|=6 k$. Consequently, the number of vertices in the $k$-th ring is $\left|R_{k}\right|=6(2 k-1)$ and so the first $m$ rings contain $\sum_{k=1}^{m}\left|R_{k}\right|=6 m^{2}$ vertices.

\section{System of Coordinates in the Hexagonal Lattice}

Suppose we have a square lattice $S$ with the usual Cartesian system of coordinates, i.e., such that the Euclidean distance of any two adjacent vertices is 1 . Using the coordinates, any vertex $v$ of this square lattice can be represented as a pair $(a, b)$ and then clearly the (graph) distance of two vertices $v_{1}=\left(a_{1}, b_{1}\right)$ and $v_{2}=\left(a_{2}, b_{2}\right)$ in the square lattice is $\operatorname{dist}_{S}\left(v_{1}, v_{2}\right)=\left|a_{1}-a_{2}\right|+\left|b_{1}-b_{2}\right|$. Removing all edges joining two vertices $(a, b)$ and $(a, b+1)$ in this square lattice whenever $a+b$ is odd, we get a lattice with hexagonal cells. Then, the orthocenter's neighbours are vertices $(0,1),(-1,0),(1,0)$, but vertex $(0,-1)$ is not adjacent to the orthocenter. We can use this system of coordinates for the vertices of the hexagonal lattice, because the change of the embedding of a graph does not influence graph's combinatorial properties.

Since the hexagonal lattice is a bipartite graph, there exist two disjoint sets $V_{0}, V_{1}$ of its vertices such that $V_{0} \cup V_{1}=V$ and each edge $u v \in E$ satisfies $u \in V_{0}$ and $v \in V_{1}$ or otherwise. Without loss of generality let orthocenter belong to $V_{0}$. Every vertex $v \in V_{0}$ is called to be of a type 0 , similarly vertices in $V_{1}$ are called of a type 1 . The type of a vertex $v$ is denoted by $\tau(v)$.

Proposition 3.1. Let $v=(a, b)$ be a vertex of the hexagonal lattice. Then the type of $v$ is

$$
\tau(v)=((a+b) \bmod 2)
$$


Lemma 3.2. Let $v_{1}=\left(a_{1}, b_{1}\right), v_{2}=\left(a_{2}, b_{2}\right)$ be two vertices of the hexagonal lattice, assume that $b_{1} \geq b_{2}$. Then the distance between $v_{1}$ and $v_{2}$ is

$$
\operatorname{dist}\left(v_{1}, v_{2}\right)= \begin{cases}\left|a_{1}-a_{2}\right|+\left|b_{1}-b_{2}\right|, & \text { if }\left|a_{1}-a_{2}\right| \geq\left|b_{1}-b_{2}\right| ; \\ 2\left|b_{1}-b_{2}\right|-\tau\left(v_{1}\right)+\tau\left(v_{2}\right), & \text { if }\left|a_{1}-a_{2}\right|<\left|b_{1}-b_{2}\right| .\end{cases}
$$

Proof. In the first case, every $v_{1} v_{2}$-path must contain at least $\left|a_{1}-a_{2}\right|$ horizontal edges and at least $\left|b_{1}-b_{2}\right|$ vertical edges. And a $v_{1} v_{2}$-path containing exactly $\left|a_{1}-a_{2}\right|+\left|b_{1}-b_{2}\right|$ edges clearly exists. Denoting $v_{3}=$ $\left(a_{2}+\left(b_{1}-b_{2}\right), b_{1}\right)$, we will investigate three possibilities for the second case:

$\alpha)$ if $\tau\left(v_{1}\right)=\tau\left(v_{2}\right)$ : the distance is $\operatorname{dist}\left(v_{1}, v_{2}\right)=\operatorname{dist}\left(v_{3}, v_{2}\right)=\mid a_{2}+\left(b_{1}-\right.$ $\left.b_{2}\right)-a_{2}|+| b_{1}-b_{2}|=2| b_{1}-b_{2} \mid$;

$\beta)$ if $\tau\left(v_{1}\right)=0, \tau\left(v_{2}\right)=1$ : the distance is $\operatorname{dist}\left(v_{1}, v_{2}\right)=\operatorname{dist}\left(v_{3}, v_{2}\right)+1=$ $\left|a_{2}+\left(b_{1}-b_{2}\right)-a_{2}\right|+\left|b_{1}-b_{2}\right|+1=2\left|b_{1}-b_{2}\right|+1$

$\gamma)$ if $\tau\left(v_{1}\right)=1, \tau\left(v_{2}\right)=0$ : the distance is $\operatorname{dist}\left(v_{1}, v_{2}\right)=\operatorname{dist}\left(v_{3}, v_{2}\right)-1=$ $\left|a_{2}+\left(b_{1}-b_{2}\right)-a_{2}\right|+\left|b_{1}-b_{2}\right|-1=2\left|b_{1}-b_{2}\right|-1$.

Corollary 3.2.1. Let $v_{1}=\left(a_{1}, b_{1}\right), v_{2}=\left(a_{2}, b_{2}\right)$ be two vertices of the hexagonal lattice, assume that $b_{1} \geq b_{2}$. Then $\operatorname{dist}\left(v_{1}, v_{2}\right) \geq 2\left|b_{1}-b_{2}\right|-1$.

Corollary 3.2.2. Let $v_{1}=\left(a_{1}, b_{1}\right), v_{2}=\left(a_{2}, b_{2}\right)$ be two vertices of the hexagonal lattice, assume that $b_{1} \geq b_{2}$. Then $\operatorname{dist}\left(v_{1}, v_{2}\right) \geq\left|a_{1}-a_{2}\right|$.

\section{Proof of the TheOrem}

In terms of the definition of Heuvel, Leese and Shepherd [6], the $n$-colorings from Lemmas 4.1, 4.4, 4.7 and 4.10 below are colorings by arithmetic progression, i.e., there exist nonnegative integers $a$ and $b$ such that $\varphi\left(m_{1}, m_{2}\right)=$ $a m_{1}+b m_{2}(\bmod n)$ for all vertices $\left(m_{1}, m_{2}\right) \in V(H)$. Here $\varphi$ is a $d$-distant $n$-coloring.

A set system $\left\{F_{i}, i \in I\right\}$, where $I$ is an index-set, is an $n$-system of color classes for a given graph $G$, or an $n$-system for brevity, if the two following conditions hold:

(i) $\left\{F_{i}, i \in I\right\}$ partitions the vertex-set of $G$,

(ii) there exists an injective assignment $\psi: I \rightarrow\{1,2, \ldots, n\}$. 
Letting $\varphi(v)=\psi(i)$ for any $i \in I$ and $v \in F_{i}$ we then get an induced $n$-coloring of the graph $G$.

To prove Theorem 1.1 we will consider four cases depending on the remainder of $d$ modulo 4 . In each case we introduce an $n$-system giving an upper bound for $\chi_{d}$ and then we show the number of colors that is necessary. By the statement $r=(a \bmod m)$ we mean that $r$ is the remainder of $a$ divided by $m$. An expression $a \equiv b(\bmod m)$ denotes that $a$ is congruent with $b$ modulo $m$.

Case 1. $d=4 m-1$ for some positive integer $m$

Lemma 4.1. Let $I=\{(i, j) ; i \in\{0,1,2, \ldots, 3 m-1\}$ and $j \in\{0,1,2, \ldots$, $2 m-1\}\}$. The set system

$$
\begin{aligned}
& F_{i, j}=\{(a, b) ;[a \equiv i \quad(\bmod 6 m) \text { and } b \equiv j \quad(\bmod 2 m)] \text { or } \\
& [a \equiv i+3 m \quad(\bmod 6 m) \text { and } b \equiv j+m \quad(\bmod 2 m)]\}
\end{aligned}
$$

for $(i, j) \in I$ is a $6 m^{2}$-system of color classes for the hexagonal lattice.

Proof. (i) Let $(x, y) \in V$. If the remainder of $x$ divided by $6 m$ is less than $3 m$, denote $i, j$ in the following way: $i=(x \bmod 6 m)$ and $j=(y$ $\bmod 2 m)$. Otherwise, denote $i=((x-3 m) \bmod 6 m)$ and $j=((y-m)$ $\bmod 2 m)$. Then $(i, j) \in I$ and $(x, y) \in F_{i, j}$. The pair $(i, j)$ is determined uniquely, and so this set system partitions the set $V$.

(ii) The assignment $\psi(i, j)=2 m i+j+1$ for $(i, j) \in I$ is injective to the set $\left\{1,2, \ldots, 6 m^{2}\right\}$.

Lemma 4.2. There is a $(4 m-1)$-distant $6 m^{2}$-coloring of the hexagonal lattice.

Proof. We will prove that the $6 \mathrm{~m}^{2}$-coloring induced by the set system in Lemma 4.1 is a $(4 m-1)$-distant coloring. Let $(i, j)$ be arbitrary but fixed member of the index-set $I$ from Lemma 4.1. Let $v_{1}, v_{2} \in F_{i, j}$ be any two distinct vertices such that $v_{1}=\left(a_{1}, b_{1}\right), v_{2}=\left(a_{2}, b_{2}\right)$ and without loss of generality let assume $b_{1} \geq b_{2}$. We have two cases:

Case (i) if $a_{1} \equiv a_{2}(\bmod 6 m)$

This implies that $b_{1} \equiv b_{2}(\bmod 2 m)$. If $a_{1} \neq a_{2}$, then, using Corollary $3.2 .2, \operatorname{dist}\left(v_{1}, v_{2}\right) \geq\left|a_{2}-a_{1}\right| \geq 6 m>4 m-1$. For the possibility that 
$a_{1}=a_{2}$ realize that vertices $v_{1}, v_{2}$ are of the same type, because there exists a positive integer $y$ such that $b_{1}=b_{2}+2 m y$. Then by Lemma 3.2 $\operatorname{dist}\left(v_{1}, v_{2}\right)=2\left|b_{2}-b_{1}\right| \geq 4 m>4 m-1$.

Case (ii) if $a_{1} \equiv a_{2}-3 m(\bmod 6 m)$

Then $b_{1} \equiv b_{2}-m(\bmod 2 m)$, i.e., there exist an integer $x$ and positive integer $y$ such that $a_{1}=a_{2}-3 m+6 m x$ and $b_{1}=b_{2}-m+2 m y$. When $x>1$ or $x<0$, from the Corollary 3.2.2 we have $\operatorname{dist}\left(v_{1}, v_{2}\right) \geq\left|a_{2}-a_{1}\right| \geq 9 m>4 m-1$. In case that $y>1$ there is $\left|b_{1}-b_{2}\right| \geq 3 \mathrm{~m}$ and from the Corollary 3.2.1 there is $\operatorname{dist}\left(v_{1}, v_{2}\right) \geq 2\left|b_{2}-b_{1}\right|-1 \geq 6 m-1>4 m-1$. The remaining possible values of $x$ are 0 and 1 , so $\left|a_{2}-a_{1}\right|=3 m$, and $y=1$, so $\left|b_{2}-b_{1}\right|=m$. This means that $\left|a_{2}-a_{1}\right| \geq\left|b_{2}-b_{1}\right|$ and finally using the Lemma 3.2 we get that $\operatorname{dist}\left(v_{1}, v_{2}\right)=\left|a_{2}-a_{1}\right|+\left|b_{2}-b_{1}\right|=4 m>4 m-1$.

We have got that any two distinct vertices from the same color class, i.e., colored with the same color, are at distance greater than $4 m-1$, hence the $6 m^{2}$-coloring induced by the set system in Lemma 4.1 is indeed a $(4 m-1)$ distant $6 \mathrm{~m}^{2}$-coloring.

Lemma 4.3. Any $(4 m-1)$-distant coloring of the hexagonal lattice uses at least $6 \mathrm{~m}^{2}$ colors.

Proof. Consider all vertices belonging the rings $R_{1}, R_{2}, \ldots, R_{m}$. The distance between any of them and the central eye is at most $2 m-2$. The greatest possible distance for two vertices within central eye is 3 , so any two of the considered vertices are of distance at most $2 \cdot(2 m-2)+3=4 m-1=d$ and so all of them must be colored with distinct colors. Thus, by Lemma 2.3 , at least $6 m^{2}$ colors are necessary.

To summarize Case $1, \chi_{d}=6 m^{2}=\frac{3}{8}(d+1)^{2}$ if $d=4 m-1$.

Case 2. $d=4 m+1$ for some nonnegative integer $m$

Lemma 4.4. Let $I=\{(i, j) ; i \in\{0,1,2, \ldots, 3 m\}$ and $j \in\{0,1,2, \ldots, 2 m\}\} \cup$ $\{(3 m+1, j) ; j \in\{m, m+1, m+2, \ldots, 2 m\}\}$. The set system

$$
\begin{aligned}
& F_{i, j}=\{(a, b) ;[a=i+s+(6 m+3) t \text { and } \\
&b=j+(2 m+1) s-t \text { for some integers } s, t] \text { or } \\
& {[a=i+s+(6 m+3) t+3 m+2 \text { and }} \\
&b=j+(2 m+1) s-t+m \text { for some integers } s, t]\}
\end{aligned}
$$


for $(i, j) \in I$ is a $\left(6 m^{2}+6 m+2\right)$-system of color classes for the hexagonal lattice.

Proof. (i) Observe that there is a pattern of colors, which is replicated over the hexagonal lattice. Any two vertices $(x, y)$ and $\left(x+6 m^{2}+6 m+2, y\right)$ are colored with the same color: Let $(x, y)$ have a color $(i, j)$ and let there exist integers $s$ and $t$ such that $x=i+s+(6 m+3) t$ and $y=j+(2 m+1) s-t$. Now $x+6 m^{2}+6 m+2=i+s+(6 m+3) t+6 m^{2}+6 m+2=i+s+$ $(6 m+3)(t+m)+3 m+2$ and $b=j+(2 m+1) s-(t+m)+m$. Since $m$ is fixed, we can denote $t^{\prime}=t+m$ and so we get that $x+6 m^{2}+6 m+2=$ $i+s+(6 m+3) t^{\prime}+3 m+2$ and $b=j+(2 m+1) s-t^{\prime}+m$, which means that the vertex $\left(x+6 m^{2}+6 m+2, y\right)$ belongs to the same color set as $(x, y)$. The remaining possibility for the vertex $(x, y)$ that there exist integers $s$ and $t$ such that $x=i+s+(6 m+3) t+3 m+2$ and $y=j+(2 m+1) s-t+m$, the proof is analogical. Similarly it holds that any two vertices $(x, y)$ and $\left(x, y+6 m^{2}+6 m+2\right)$ are colored with the same color. Therefore, a "square" pattern containing $\left(6 m^{2}+6 m+2\right)^{2}$ vertices is replicated throughout the lattice.

When we focus on any "row" (or "column") of this pattern, we can see that each of the $6 m^{2}+6 m+2$ vertices belongs to different color set from our set system. A stable order of colors is repeated there. For example, it is easy to check that the vertices $(x, 0)$ for integers $x \in\left(0,6 m^{2}+6 m+1\right)$ belong to these respective color sets: $(0,0),(1,0), \ldots,(3 m, 0),(0, m+1)$, $(1, m+1), \ldots,(3 m+1, m+1), \ldots,(0, m),(1, m), \ldots,(3 m+1, m)$. The same sequence is repeated in each row, just starting with a different element of the sequence. Thus, the set system $F_{i, j}$ partitions the vertex set $V$.

(ii) Let $\delta_{p, r}$ be Kronecker's Delta, i.e., a function which assigns $\delta_{p, r}=1$, if $p=r$ and $\delta_{p, r}=0$, if $p \neq r$. Let $\psi(i, j)=(2 m+1) i+j+1-m \delta_{3 m+1, i}$ be for any $(i, j) \in I$. Since $j$ gets values from the set $\{0,1,2, \ldots, 2 m\}$, the assignment $\psi(i, j)$ is injective to the set $\left\{1,2, \ldots, 6 m^{2}+6 m+2\right\}$.

Lemma 4.5. There is a $(4 m+1)$-distant $\left(6 m^{2}+6 m+2\right)$-coloring of the hexagonal lattice.

Proof. We will prove that the $\left(6 m^{2}+6 m+2\right)$-coloring induced by the set system from Lemma 4.4 is a $(4 m+1)$-distant coloring. Let $(i, j)$ be arbitrary but fixed member of the index-set $I$ from Lemma 4.4. Let $v_{1}, v_{2} \in F_{i, j}$ be any two distinct vertices such that $v_{1}=\left(a_{1}, b_{1}\right), v_{2}=\left(a_{2}, b_{2}\right)$ and without loss of generality let $b_{1} \geq b_{2}$. 
When we show that $\left|a_{1}-a_{2}\right|>4 m+1$, the Corollary $3.2 .2 \operatorname{implies} \operatorname{dist}\left(v_{1}, v_{2}\right)$ $\geq\left|a_{1}-a_{2}\right|>4 m+1$. And when we show that $\left|b_{1}-b_{2}\right|>2 m+1$, then the Corollary 3.2.1 implies $\operatorname{dist}\left(v_{1}, v_{2}\right) \geq 2\left|b_{1}-b_{2}\right|-1>4 m+1$. We have to analyse four cases:

Case (i). There exist integers $x_{1}, x_{2}, y_{1}, y_{2}$ such that

$$
\begin{aligned}
& a_{1}=i+x_{1}+(6 m+3) y_{1}, b_{1}=j+(2 m+1) x_{1}-y_{1}, \\
& a_{2}=i+x_{2}+(6 m+3) y_{2}, \quad b_{2}=j+(2 m+1) x_{2}-y_{2} .
\end{aligned}
$$

Hence there exist integers $x=x_{1}-x_{2}, y=y_{1}-y_{2}$ such that $a_{1}-a_{2}=$ $x+(6 m+3) y$ and $b_{1}-b_{2}=(2 m+1) x-y$, and not both equal zero (otherwise $v_{1}=v_{2}$ ). When $y=0$, for $|x|>1$ there is $\left|b_{1}-b_{2}\right|>2 m+1$. For the case that $|x|=1$ we have $\left|b_{1}-b_{2}\right|=2 m+1$ and $\left|a_{1}-a_{2}\right|=1$, and the Lemma 3.2 follows $\operatorname{dist}\left(v_{1}, v_{2}\right)=2\left|b_{1}-b_{2}\right|=4 m+2>4 m+1$.

Let now $y>0$. For $x \geq 0$ there is $\left|a_{1}-a_{2}\right| \geq 6 m+3>4 m+1$. Since $b_{1} \geq b_{2}, x$ cannot be negative. Finally, let $y<0$. For $x \leq 0$ there is $\left|a_{1}-a_{2}\right| \geq 6 m+3>4 m+1$. When $x>0$ we have $\left|b_{1}-b_{2}\right|>2 m+1$.

Case (ii). There exist integers $x_{1}, x_{2}, y_{1}, y_{2}$ such that

$$
\begin{aligned}
& a_{1}=i+x_{1}+(6 m+3) y_{1}+3 m+2, b_{1}=j+(2 m+1) x_{1}-y_{1}+m, \\
& a_{2}=i+x_{2}+(6 m+3) y_{2}+3 m+2, b_{2}=j+(2 m+1) x_{2}-y_{2}+m .
\end{aligned}
$$

But this case can be transformed to Case (i).

Case (iii). There exist integers $x_{1}, x_{2}, y_{1}, y_{2}$ such that

$$
\begin{aligned}
& a_{1}=i+x_{1}+(6 m+3) y_{1}, b_{1}=j+(2 m+1) x_{1}-y_{1}, \\
& a_{2}=i+x_{2}+(6 m+3) y_{2}+3 m+2, b_{2}=j+(2 m+1) x_{2}-y_{2}+m .
\end{aligned}
$$

Hence there are integers $x=x_{1}-x_{2}, y=y_{1}-y_{2}$ such that $a_{1}-a_{2}=$ $x+(6 m+3) y-3 m-2$ and $b_{1}-b_{2}=(2 m+1) x-y-m$. For $x=y=0$ we have $\left|a_{1}-a_{2}\right|=3 m+2$ and $\left|b_{1}-b_{2}\right|=m$. Similarly, for $x=1$ and $y=0$ we have $\left|a_{1}-a_{2}\right|=3 m+1$ and $\left|b_{1}-b_{2}\right|=m+1$. Finally for $x=1$ and $y=1$ there is $\left|a_{1}-a_{2}\right|=3 m+2$ and $\left|b_{1}-b_{2}\right|=m$. Thus in all three cases according to Lemma 3.2 there holds that $\operatorname{dist}\left(v_{1}, v_{2}\right)=\left|a_{1}-a_{2}\right|+\left|b_{1}-b_{2}\right|=$ $4 m+2>4 m+1$. The case $x=0, y=1$ cannot occur due to assumption that $b_{1} \geq b_{2}$. 
For $x \geq 2$ and $y=1$ or $y=0$ there is $\left|b_{1}-b_{2}\right| \geq 3 m+1>2 m+1$. Assuming $x \geq 0, y \geq 0$ (except already investigated cases) we have $\left|a_{1}-a_{2}\right| \geq 9 m+4>$ $4 m+1$. The case $x<0, y \geq 0$ cannot occur due to assumption that $b_{1} \geq b_{2}$.

Let now $y \leq-1$. The subcase when $x \leq 1$ satisfies $\left|a_{1}-a_{2}\right| \geq 9 m+4>$ $4 m+1$. For the subcase $x \geq 2$ we have $\left|b_{1}-b_{2}\right| \geq 3 m+3>2 m+1$.

Case (iv). There exist integers $x_{1}, x_{2}, y_{1}, y_{2}$ such that

$$
\begin{aligned}
& a_{1}=i+x_{1}+(6 m+3) y_{1}+3 m+2, b_{1}=j+(2 m+1) x_{1}-y_{1}+m, \\
& a_{2}=i+x_{2}+(6 m+3) y_{2}, b_{2}=j+(2 m+1) x_{2}-y_{2} .
\end{aligned}
$$

Hence there exist integers $x=x_{1}-x_{2}+1, y=y_{1}-y_{2}+1$ such that $a_{1}-a_{2}=x+(6 m+3) y-3 m-2$ and $b_{1}-b_{2}=(2 m+1) x-y-m$. But this is the same as in Case (iii).

We have got that any two distinct vertices from the same color class, i.e., colored with the same color, are at distance greater than $4 m+1$, hence the $\left(6 m^{2}+6 m+2\right)$-coloring induced by the set system from Lemma 4.4 is indeed a $(4 m+1)$-distant $\left(6 m^{2}+6 m+2\right)$-coloring.

Lemma 4.6. Any $(4 m+1)$-distant coloring of the hexagonal lattice uses at least $6 m^{2}+6 m+2$ colors.

Proof. Consider all vertices belonging to the rings $R_{1}, R_{2}, \ldots, R_{m}$ and the set $R_{m+1}^{-}$. Similarly to the proof of Lemma 4.3, one can see that at least $6 m^{2}+6 m$ colors are necessary. Let $H^{\prime}$ be the graph with vertex-set $R_{m+1}^{+}$ and where two vertices $u, v$ are adjacent in $H^{\prime}$ if and only if they are at distance 2 in $H$. This graph has six components $C_{1}, C_{2}, \ldots, C_{6}$, each of which is a path with $m+1$ vertices.

The distance in $H$ between any two vertices of the $V\left(C_{1}\right)$ is at most $2 m$, hence they must be colored with distinct colors. The effort to use only $6 m^{2}+6 m$ already used colors fails, because there are at most $m$ vertices such that their colors may be from already used. Hence we need at least one new color. This is true for any component of $H^{\prime}$. Clearly, there are two components $C_{i}, C_{j}(i, j \in\{1,2, \ldots, 6\}, i \neq j)$ such that the distance between any two of their vertices is not more than $2 m+2 m+1$, thus their new colors must be distinct and we have already used $6 m^{2}+6 m+2$ colors.

To summarize Case 2, $\chi_{d}=6 m^{2}+6 m+2=\frac{3}{8}(d+1)^{2}+\frac{1}{2}$ if $d=4 m+1$.

Case 3. $d=4 m+2$ for some nonnegative integer $m$-an upper bound. 
Lemma 4.7. Let $I=\{(i, j) ; i \in\{0,1,2, \ldots, 6 m+3\}, j \in\{0,1,2, \ldots$, $2 m+1\}$, and $i+j \equiv 0(\bmod 2)\}$. The set system

$$
\begin{aligned}
& F_{i, j}=\{(a, b) ;[a \equiv i \quad(\bmod (6 m+4)) \text { and } b \equiv j \quad(\bmod (2 m+2))] \text { or } \\
& [a \equiv i-3 m-2 \quad(\bmod (6 m+4)) \text { and } b \equiv j+m+1 \quad(\bmod (2 m+2))]\}
\end{aligned}
$$

for $(i, j) \in I$ is a $\left(6 m^{2}+10 m+4\right)$-system of color classes for the hexagonal lattice.

Proof. (i) Let $(x, y) \in V$. If the sum $x+y$ is even, denote $i, j$ in the following way: $i=(x \bmod (6 m+4))$ and $j=(y \bmod (2 m+2))$. Otherwise, denote $i=((x+3 m+2) \bmod (6 m+4))$ and $j=((y-m-1) \bmod (2 m+2))$. Thus, $i+j$ is even, so $(i, j) \in I$ and $(x, y) \in F_{i, j}$. The pair $(i, j)$ is determined uniquely, and so this set system partitions the set $V$.

(ii) It is easy to check that the assignment $\psi(i, j)=(m+1) i+\left[\frac{j+1}{2}\right]$ for $(i, j) \in I$ is injective to the set $\left\{1,2, \ldots, 6 m^{2}+10 m+4\right\}$.

Lemma 4.8. There is a $(4 m+2)$-distant $\left(6 m^{2}+10 m+4\right)$-coloring of the hexagonal lattice.

Proof. If $m=0$, any two vertices colored with the same color by the 4-coloring induced by the 4 -system from Lemma 4.7 are at distance more than 2, hence this 4-coloring is 2-distant. Now, assume that $m>0$.

We will prove that the $\left(6 m^{2}+10 m+4\right)$-coloring induced by the set system from Lemma 4.7 is a $(4 m+2)$-distant coloring. Let $(i, j)$ be arbitrary but fixed member of the index-set $I$ from Lemma 4.7. Let $v_{1}, v_{2} \in F_{i, j}$ be any two distinct vertices such that $v_{1}=\left(a_{1}, b_{1}\right), v_{2}=\left(a_{2}, b_{2}\right)$ and, without loss of generality, $b_{1} \geq b_{2}$. There are 4 cases:

\section{Case (i) $\tau\left(v_{1}\right)=0, \tau\left(v_{2}\right)=0$}

This means that $a_{1} \equiv a_{2}(\bmod (6 m+4))$ and $b_{1} \equiv b_{2}(\bmod (2 m+2))$, i.e., there are an integer $x$ and a nonnegative integer $y$ such that $a_{1}=$ $a_{2}+x(6 m+4), b_{1}=b_{2}+y(2 m+2)$ and not both equal zero. When $y>0$, then by the Corollary 3.2.1 $\operatorname{dist}\left(v_{1}, v_{2}\right) \geq 2\left(b_{1}-b_{2}\right)-1 \geq 2(2 m+2)-1=$ $4 m+3>4 m+2$. When $y=0$ we have $b_{1}=b_{2}$ and since $x \neq 0$, by Lemma 3.2 is then $\operatorname{dist}\left(v_{1}, v_{2}\right)=\left|a_{2}-a_{1}\right|=|x|(6 m+4) \geq 6 m+4>4 m+2$. 
Case (ii) $\tau\left(v_{1}\right)=1, \tau\left(v_{2}\right)=1$

This is the situation when it holds that $a_{1}-3 m-2 \equiv a_{2}-3 m-2(\bmod (6 m+$ $4)$ ) and that $b_{1}+m+1 \equiv b_{2}+m+1(\bmod (2 m+2))$, which is the same situation as in Case (i).

Case (iii) $\tau\left(v_{1}\right)=0, \tau\left(v_{2}\right)=1$

We have $a_{1} \equiv a_{2}+3 m+2(\bmod (6 m+4))$ and $b_{1} \equiv b_{2}-m-1(\bmod (2 m+2))$, thus there are an integer $x$ and a positive integer $y$ such that $a_{1}=a_{2}+3 m+$ $2+x(6 m+4), b_{1}=b_{2}-m-1+y(2 m+2)$. When $x>0$ or $x<-1$, then by Corollary 3.2.2 $\operatorname{dist}\left(v_{1}, v_{2}\right) \geq\left|a_{1}-a_{2}\right| \geq 9 m+6>4 m+2$. Having $x=-1$ or $x=0$ there is $\left|a_{2}-a_{1}\right|=3 m+2$. If $y>1$, using Corollary 3.2.1 we have $\operatorname{dist}\left(v_{1}, v_{2}\right) \geq 2(3 m+3)-1>4 m+2$. Finally if $y=1$, we have $\left|b_{1}-b_{2}\right|=$ $m+1$, and so by Lemma 3.2 we have $\operatorname{dist}\left(v_{1}, v_{2}\right)=3 m+2+m+1>4 m+2$.

Case (iv) $\tau\left(v_{1}\right)=1, \tau\left(v_{2}\right)=0$

Now $a_{1}+3 m+2 \equiv a_{2}(\bmod (6 m+4))$ and $b_{1}-m-1 \equiv b_{2}(\bmod (2 m+2))$, so there are an integer $x$ and a nonnegative integer $y$ such that $a_{1}=a_{2}-$ $3 m-2+x(6 m+4), b_{1}=b_{2}+m+1+y(2 m+2)$. By the substitution $\bar{x}=x-1, \bar{y}=y+1$ this case is transformed to Case (iii).

We have got that any two distinct vertices from the same color class, i.e., colored with the same color, are at distance greater than $4 m+2$, hence the $\left(6 m^{2}+10 m+4\right)$-coloring induced by the set system from Lemma 4.7 is indeed a $(4 m+2)$-distant $\left(6 m^{2}+10 m+4\right)$-coloring.

To summarize Case $3, \chi_{d} \leq 6 m^{2}+10 m+4=\frac{3}{8}\left(d+\frac{4}{3}\right)^{2}-\frac{1}{6}$ if $d=4 m+2$.

Case 4. $d=4 m$ for some positive integer $m$-an upper bound

Lemma 4.9. Let $I=\{(i, j) ; i \in\{0,1,2, \ldots, 6 m\}, j \in\{0,1,2, \ldots, 2 m\}$, and $i+j \equiv 0(\bmod 2)\}$. The set system

$$
\begin{aligned}
& F_{i, j}=\{(a, b) ;[a=i+s-(6 m+1) t \text { and } \\
&b=j+(2 m+1) s+t \text { for some integers } s, t] \text { or } \\
& {[a=i+s-(6 m+1) t+3 m+1 \text { and }} \\
&b=j+(2 m+1) s+t+m \text { for some integers } s, t]\}
\end{aligned}
$$

for $(i, j) \in I$ is a $\left(6 m^{2}+4 m+1\right)$-system of color classes for the hexagonal lattice. 
Proof. (i) Let $(x, y) \in V$. We need to find a pair $(i, j) \in I$ such that $(x, y) \in$ $F_{i, j}$. Denote $x^{\prime}=\left(x \bmod \left(12 m^{2}+8 m+2\right)\right), y^{\prime}=\left(y \bmod \left(12 m^{2}+8 m+2\right)\right)$. If we find a pair $\left(i^{\prime}, j^{\prime}\right)$ such that $\left(x^{\prime}, y^{\prime}\right) \in F_{i^{\prime}, j^{\prime}}$, then also $(x, y) \in F_{i^{\prime}, j^{\prime}}$. Because $s^{\prime} \equiv s\left(\bmod \left(12 m^{2}+8 m+2\right)\right)$ and $t^{\prime} \equiv t\left(\bmod \left(12 m^{2}+8 m+2\right)\right)$ must hold. We assign the pair $\left(i^{\prime}, j^{\prime}\right)$ to the vertex $\left(x^{\prime}, y^{\prime}\right)$ uniquely (the proof that this is always possible is not difficult but technically pretentious, therefore we omit it). Hence the set system partitions the set $V$.

(ii) Assignment $\psi(i, j)=m i+\left[\frac{i+j+1}{2}\right]$ for $(i, j) \in I$ is injective to the set $\left\{1,2, \ldots, 6 m^{2}+4 m+1\right\}$.

Lemma 4.10. There is a $4 m$-distant $\left(6 m^{2}+4 m+1\right)$-coloring of the hexagonal lattice.

Proof.The proof, that the $\left(6 m^{2}+4 m+1\right)$-coloring induced by set system from Lemma 4.9 is a $4 m$-distant coloring, is analogous to the proof of Lemma 4.8 and we omit it.

Hence $\chi_{d} \leq 6 m^{2}+4 m+1=\frac{3}{8}\left(d+\frac{4}{3}\right)^{2}+\frac{1}{3}$ for this case.

\section{Lower bounds in Case 3 and Case 4.}

The following lemma will be useful for our final result given in Lemma 4.12, which provides a lower bound on $d$-distant chromatic number for even $d$.

Lemma 4.11. Let $v_{0}$ be any vertex of $H$ and let $i$ be a positive integer. Denote $V_{i}=\left\{v \in V ; \operatorname{dist}\left(v, v_{0}\right)=i\right\}$. Then, $\left|V_{i}\right|=3 i$.

Proof. We proceed by induction on distance $i$.

$1^{\circ}$ The set $V_{1}$ contains just the neighbours of the vertex $v_{0}$, there are three of them. The set $V_{2}$ contains 6 vertices, which are neighbouring to the vertices of $V_{1}$ and different from $v_{0}$.

$2^{\circ}$ Let $j \geq 3$ be an integer. Suppose that for all $i=1,2, \ldots, j$ it holds that $\left|V_{i}\right|=3 i$. Look at the set $V_{j+1}$. It contains all the neighbours of the vertices belonging to $V_{j}$ such that does not belong to $V_{j-1}$. Each vertex belonging to $V_{j}$ has 3 neighbours, so we have $3 \cdot 3 j=9 j$ possible candidates. Note that for each vertex $v_{x} \in V_{j}$ there exist vertices $v_{y}, v_{z} \in V_{j}$ such that $\operatorname{dist}\left(v_{x}, v_{y}\right)=\operatorname{dist}\left(v_{x}, v_{z}\right)=2$. Therefore, precisely $\left|V_{j}\right|$ vertices are counted twice; we are left with $9 j-3 j=6 j$ candidates. Those still contain the vertices belonging to $V_{j-1}$, there are $3 j-3$ such vertices. Finally, we obtain $6 j-(3 j-3)=3 j+3$ vertices which belong to $V_{j+1}$. 
To avoid collision with the previous notation, consider here that $d=2 p$ for some integer $p \geq 2$.

Lemma 4.12. Any $2 p$-distant coloring of the hexagonal lattice requires at least $\frac{3}{2} p^{2}+\frac{3}{2} p+2$ colors.

Proof. Let $v_{0}$ be any vertex of $H$ and denote $V_{i}$ for $i=1,2, \ldots, p$ as in Lemma 4.11. Consider all the vertices that belong to the sets $V_{1}, V_{2}, \ldots, V_{p}$ (denote their union as $W$ ), their number is $\sum_{i=1}^{p} 3 i=3 \frac{p(p+1)}{2}$. Any vertex of $W$ is at most at distance $p$ from the vertex $v_{0}$, hence mutual distance of any pair of the vertices belonging to $W$ is at most $2 p=d$. This implies that all of them, including the vertex $v_{0}$, have to be colored by distinct colors, therefore at least $\frac{3}{2} p^{2}+\frac{3}{2} p+1$ colors are necessary for any $2 p$-distant coloring.

Nevertheless, another color is enforced if we look at the set $V_{p+1}$. Let $v_{1} \in V_{p+1}$ and $v_{2} \in V_{r}$ such that $r \leq p-1$ (or $\left.v_{2}=v_{0}\right)$. Since $\operatorname{dist}\left(v_{1}, v_{2}\right) \leq$ $2 p$, these vertices cannot use the same color. In order to come to a contradiction, suppose that $\frac{3}{2} p^{2}+\frac{3}{2} p+1$ is a sufficient number of colors.

Thus, the vertices that belong to $V_{p+1}$ can be colored only by colors used for the vertices in $V_{p}$. There are only $\left|V_{p}\right|=3 p$ of such colors, whereas we need to color $\left|V_{p+1}\right|=3 p+3$ vertices. Observe that there cannot exist a color used at three (or more) vertices from the set $V_{p+1}$, as it must also color one vertex from $W$. By the pigeonhole principle there exist at least three colors such that each of them colors precisely one pair of vertices from the set $V_{p+1}$ and one vertex from the set $W$. Moreover, we already know from the previous paragraph that the latter must belong to $V_{p}$.

If we try to find such a triple of vertices of distances greater than $d$, we will find it very restricted. A choice of a vertex from $V_{p}$ which is not a corner (with the meaning as in Proposition 2.2) exhibits that at distance at least $d+1$ there are in $V_{p+1}$ only vertices whose mutual distance is lower than $d$. So we have to consider a corner vertex from the set $V_{p}$. If we fix that corner vertex, there exist a unique pair of vertices belonging to $V_{p+1}$ at pairwise distance greater than $d$. So we have a triple of vertices where the same color can be made use of. We can also find another such triple which does not coincide with the first triple. However, it is not possible to find one more triple, because the triples are enforced uniquely and their "shape" implies a coincidence.

Therefore, at least one vertex from the set $V_{p+1}$ is left free and so, at least one more color is necessary. 
We conjecture that any $(4 m+2)$-distant coloring of the hexagonal lattice uses at least $6 m^{2}+10 m+4$ colors.

This is true for the cases when $m=0$ or 1 . Consider any vertex and its three neighbours. Their mutual distances are not greater than 2 , so they must be colored by four different colors. Thus, any 2-distant coloring of the hexagonal lattice uses at least 4 colors.

For $m \geq 1$ by Lemma 4.12 any $(4 m+2)$-distant coloring of the hexagonal lattice uses at least $6 m^{2}+9 m+5$ colors. This bound is lower than our conjectured value by $m-1=\frac{d}{4}-\frac{3}{2}$, that is, provides the same value for 6 distant coloring; uses one color less than hypothesized for 10-distant coloring; etc. However, using a computer routine that explores all possible colorings we can show that there is a subgraph of $H$ containing 109 vertices such that it requires 47 different colors for 10-distant coloring, which is one more than predicts Lemma 4.12, whereas equals to the conjectured value.

Analogously, we conjecture that any $4 \mathrm{~m}$-distant coloring of the hexagonal lattice requires at least $6 m^{2}+4 m+1$ colors.

We proved in Lemma 4.12 that any $4 m$-distant coloring of the hexagonal lattice uses at least $6 m^{2}+3 m+2$ colors. This bound is lower than our conjectured value by $m-1=\frac{d}{4}-1$, that is, provides the same value for 4 distant coloring, uses one color less than hypothesized for 8-distant coloring; etc. However, using a computer routine that explores all possible colorings we can show that there is a subgraph of $H$ containing 109 vertices such that it requires 33 different colors for 8-distant coloring, which is one more than predicts Lemma 4.12, whereas equals to the hypothesized value.

\section{Acknowledgement}

This work was supported by Science and Technology Assistance Agency under the contract No. APVT-20-004104. Support of the Slovak VEGA Grant 1/0424/03 is also acknowledged.

\section{REFERENCES}

[1] G.J. Chang and D. Kuo, The L(2,1)-labeling problem on graphs, SIAM J. Discrete Math. 9 (1996) 309-316.

[2] G. Fertin, E. Godard and A. Raspaud, Acyclic and k-distance coloring of the grid, Information Processing Letters 87 (2003) 51-58.

[3] J.P. Georges and D.M. Mauro, Generalized vertex labelings with a condition at distance two, Congr. Numer. 109 (1995) 141-159. 
[4] J.R. Griggs and R.K. Yeh, Labelling graphs with a condition at distance 2, SIAM J. Discrete Math. 5 (1992) 586-595.

[5] W.K. Hale, Frequency assignment: theory and applications, Proc. IEEE 68 (1980) 1497-1514.

[6] J. van den Heuvel, R.A. Leese and M.A. Shepherd, Graph labeling and radio Channel assignment, J. Graph Theory 29 (1998) 263-283.

[7] J. van den Heuvel and S. McGuinness, Coloring the square of a planar graph, J. Graph Theory 42 (2003) 110-124.

[8] S. Jendrol' and Z. Skupień, Local structures in plane maps and distance colourings, Discrete Math. 236 (2001) 167-177.

[9] T.R. Jensen and B. Toft, Graph Coloring Problems (John-Wiley \& Sons, New York, 1995).

[10] F. Kramer and H. Kramer, Ein farbungsproblem der knotenpunkte eines graphen bezuglich der distanz, P. Rev. Roumaine Math. Pures Appl. 14 (1969) 1031-1038.

[11] V.H. MacDonald, The cellular concept, Bell System Technical Journal 58 (1979) 15-41.

[12] C. McDiarmid and B. Reed, Colouring proximity graphs in the plane, Discrete Math. 199 (1999) 123-137.

[13] A. Ševčíková, Distant Chromatic Number of the Planar Graphs (Manuscript, P.J. Šafárik University, 2001).

[14] G. Wegner, Graphs with given Diameter and a Colouring Problem (Preprint, University of Dortmund, 1977).

Received 25 November 2003

Revised 22 February 2005 